\title{
BICOVARIANT DIFFERENTIAL CALCULI FOR FINITE GLOBAL QUOTIENTS
}

\author{
DAVID N. PHAM \\ City University of New York, USA
}

\begin{abstract}
Let $(M, G)$ be a finite global quotient, that is, a finite set $M$ with an action by a finite group $G$. In this note, we classify all bicovariant first order differential calculi (FODCs) over the weak Hopf algebra $\mathbb{k}(G \ltimes M) \simeq \mathbb{k}[G \ltimes M]^{*}$, where $G \ltimes M$ is the action groupoid associated to $(M, G)$, and $\mathbb{k}[G \ltimes M]$ is the groupoid algebra of $G \ltimes M$. Specifically, we prove a necessary and sufficient condition for a FODC over $\mathbb{k}(G \ltimes M)$ to be bicovariant and then show that the isomorphism classes of bicovariant FODCs over $\mathbb{k}(G \ltimes M)$ are in one-to-one correspondence with subsets of a certain quotient space.
\end{abstract}

\section{INTRODUCTION}

It is a somewhat lesser known fact that the differential geometry of a manifold $M$ is completely encoded by $C^{\infty}(M)$, the commutative algebra of smooth functions on $M$ ([19]). Consequently, one can discard the manifold $M$ and express any differential geometric property or object associated to $M$ algebraically in terms of the commutative algebra $C^{\infty}(M)$. Interestingly, it turns out that many of the familiar objects of differential geometry can still be defined if one replaces $C^{\infty}(M)$ by any unital associative algebra $A$, even if the latter is noncommutative. This algebraic generalization of classical differential geometry is noncommutative (differential) geometry $([7,8,23,16$, 15]). If the algebra $A$ is noncommutative, then $A$ certainly cannot be the algebra of functions on any classical manifold or space. In this case, it is helpful, for conceptual reasons, to regard $A$ as the algebra of functions on a noncommutative manifold, even though the latter does not actually exist.

2010 Mathematics Subject Classification. 58B32, 16T05, 18B40.

Key words and phrases. Global quotients, noncommutative differential geometry, first order differential calculi, weak Hopf algebras. 
There are several approaches to noncommutative geometry. The most prominent is the approach put forward by Alain Connes $([7,8])$ which is based on the idea of the spectral triple as the operator-algebraic generalization of a closed Riemannian spin manifold. However, there is another approach of significant interest: the so-called quantum group approach to noncommutative geometry. This approach originated with the work of Stanislaw Woronowicz in [23] and was developed extensively by Shahn Majid and others ([4, 2, 17, $18,11])$. In this approach, quantum groups (i.e. Hopf algebras) serve as a rich class of examples of noncommutative geometry in much the same way that Lie groups provide important examples of manifolds in classical differential geometry. In addition, the mathematical structure of quantum groups also provides one with the noncommutative geometric analogue of the classical notion of a group action.

Given an algebra $A$ (which we regard as the algebra of functions on a manifold), the starting point in the quantum group approach is a choice of first order differential calculus (FODC) over $A$; the latter is an algebraic generalization of the space of differential 1-forms on a (compact) manifold. Only after fixing a FODC can one begin to construct the algebraic analogues of other classical differential geometric objects (e.g. higher differential forms, de Rham cohomology, principle bundles, Riemannian metrics $([4,18,20]))$. Hence, the notion of a FODC plays a central role in this approach. Since the number of FODCs can be quite large, it is only natural to look for some mechanism that will filter out all but the most interesting FODCs. When $A$ is a Hopf algebra, one has such a mechanism: bicovariance. In other words, one discards all the FODC over $A$ except those which are compatible with the natural bicomodule structure of $A$ ([23]).

In this paper, we layout the groundwork for the application of noncommutative geometry (specifically the quantum group approach) to finite global quotients. Formally, a global quotient is a pair $(M, G)$ where $M$ is a manifold and $G$ is a finite group which acts on $M$; a finite global quotient then refers to the special case where the manifold $M$ is a finite set. Given a finite global quotient $(M, G)$, we take the weak Hopf algebra $\mathbb{k}(G \ltimes M) \simeq \mathbb{k}[G \ltimes M]^{*}$ as the algebra of functions associated to $(M, G)$ and classify all bicovariant FODCs over $\mathbb{k}(G \ltimes M)$. Here, $G \ltimes M$ denotes the action groupoid associated to $(M, G)$ (where we assume a left action of $G$ on $M$ ) and $\mathbb{k}[G \ltimes M]$ is the groupoid algebra of $G \ltimes M$.

The notion of bicovariant FODCs was originally defined for FODCs over a Hopf algebra $A$. However, the same basic definition also works if $A$ is replaced by a weak Hopf algebra. The problem of bicovariant differential calculi over weak Hopf algebras was originally studied in [6], especially in regard to differential calculi over weak smash products, and later again in [24]. However, the classification problem for bicovariant differential calculi over the weak Hopf algebra $\mathbb{k}(G \ltimes M)$ has yet to be studied. The current paper fills this 
gap in the literature. As one might expect, there are differences between the Hopf and weak Hopf cases. One such difference is that the universal FODC over a weak Hopf algebra is not necessarily bicovariant. This is in contrast to the Hopf algebra case where the universal FODC over any Hopf algebra is always bicovariant $([23,2])$.

Given a (general) global quotient $(X, G)$, one can associate to it an algebraic structure called a $G$-Frobenius algebra (or crossed $G$-algebra), see $[22,13]$. Specifically, one obtains a $G$-Frobenius algebra from $(X, G)$ by computing its stringy cohomology or stringy K-theory $([12,14,10])$. Hence, global quotients serve as a reservoir for $G$-Frobenius algebras. Roughly speaking, a $G$-Frobenius algebra is a $G$-graded algebra which is equipped with a Frobenius form and a $G$-action which satisfy a number of conditions. From the work of Turaev ([22]), part of the significance of $G$-Frobenius algebras comes from the fact that they are the algebraic structures which classify certain 2 -dimensional homotopy quantum field theories. Hence, $G$-Frobenius algebras can be viewed as a generalization of the commutative Frobenius algebras arising from 2-dimensional topological quantum field theories ([1]). In light of the link between global quotients and $G$-Frobenius algebras, the current paper may ultimately serve as a first step towards applying noncommutative geometry to $G$-Frobenius algebras. This possibility serves as a secondary motivation for the current paper.

The rest of the paper is organized as follows. In Sections 2 and 3, we give a fairly self contained review of the necessary background material. Specifically, in Section 2, we recall the notion of a weak Hopf algebra ([3]) as well as the weak Hopf structure of $\mathbb{k}(G \ltimes M)$. In addition, we also review some of the basic definitions and results for FODCs. In Section 3, we give a review of the FODC for finite sets $([9,8])$. Lastly, in Section 4 , we prove a necessary and sufficient condition for a FODC over $\mathbb{k}(G \ltimes M)$ to be bicovariant. This result is then used to show that the isomorphism classes of bicovariant FODCs over $\mathbb{k}(G \ltimes M)$ are in one-to-one correspondence with subsets of a certain quotient space. In the special case where $M$ is a point, our result reduces to the well known fact that the isomorphism classes of bicovariant FODCs over the Hopf algbera $\mathbb{k}(G) \simeq \mathbb{k}[G]^{*}$ are in one-to-one correspondence with subsets of the set of non-identity conjugacy classes of $G([5])$.

\section{Preliminaries}

In this section, we recall some of the relevant background for the paper. Unless stated otherwise, we use the following notation for the rest of the paper:

- $\mathbb{k}$ is a field of characteristic 0 ,

- $\otimes:=\otimes_{\mathbb{k}}$,

- $e$ is the identity element of a group. 
All algebras are unital associative $\mathbb{k}$-algebras unless stated otherwise. The coproduct $\Delta$ of a colagebra $C$ will be expressed in Sweedler notation ([21]) as

$$
\Delta(c)=c_{(1)} \otimes c_{(2)}, \quad(\Delta \otimes i d) \circ \Delta(c)=(i d \otimes \Delta) \circ \Delta(c)=c_{(1)} \otimes c_{(2)} \otimes c_{(3)} .
$$

Likewise, a left (right) coaction $\Delta_{L}\left(\Delta_{R}\right)$ of $C$ on a vector space $V$ will be denoted as

$$
\Delta_{L}(c)=c_{(-1)} \otimes c_{(0)} \in C \otimes V, \quad \Delta_{R}(c)=c_{(0)} \otimes c_{(1)} \in V \otimes C .
$$

2.1. Weak Hopf algebras.

Definition 2.1. A weak Hopf algebra over $\mathbb{k}$ consists of the following data:

$$
(H, \mu, 1, \Delta, \varepsilon, S)
$$

where

(a) $H$ is an associative algebra over $\mathbb{k}$ with product

$$
\mu: H \otimes H \rightarrow H, \quad x \otimes y \mapsto x y
$$

and unit element 1.

(b) $H$ is a coassociative colagebra over $\mathbb{k}$ with coproduct

$$
\Delta: H \rightarrow H \otimes H, \quad x \mapsto x_{(1)} \otimes x_{(2)}
$$

and counit $\varepsilon: H \rightarrow \mathbb{k}$.

which satisfy the following conditions:

(i) the algebra and coalgebra structures satisfy the following compatibility relations:

multiplicativity of the coproduct: for all $x, y \in H$

$$
\Delta(x y)=\Delta(x) \Delta(y),
$$

weak mulitplicativity of the counit: for all $x, y, z \in H$

$$
\varepsilon(x y z)=\varepsilon\left(x y_{(1)}\right) \varepsilon\left(y_{(2)} z\right)=\varepsilon\left(x y_{(2)}\right) \varepsilon\left(y_{(1)} z\right),
$$

weak comultiplicativity of the unit:

$$
\begin{aligned}
& \left(\Delta \otimes i d_{H}\right) \circ \Delta(1)=(\Delta(1) \otimes 1)(1 \otimes \Delta(1)), \\
& \left(\Delta \otimes i d_{H}\right) \circ \Delta(1)=(1 \otimes \Delta(1))(\Delta(1) \otimes 1) .
\end{aligned}
$$

(ii) the linear map $S: H \rightarrow H$ (called the antipode) satisfies the following conditions for all $x \in H$ :

$$
\begin{aligned}
& x_{(1)} S\left(x_{(2)}\right)=\varepsilon\left(1_{(1)} x\right) 1_{(2)}, \\
& S\left(x_{(1)}\right) x_{(2)}=1_{(1)} \varepsilon\left(x 1_{(2)}\right), \\
& S\left(x_{(1)}\right) x_{(2)} S\left(x_{(3)}\right)=S(x) .
\end{aligned}
$$

REMARK 2.2. Naturally, every Hopf algebra is a weak Hopf algebra. For a weak Hopf algebra $H$, one has the following equivalent statements:

(a) $H$ is a Hopf algebra, 
(b) $\Delta(1)=1 \otimes 1$,

(c) $\varepsilon(x y)=\varepsilon(x) \varepsilon(y)$ for all $x, y \in H$.

For the sake of completeness, we recall the notion of a groupoid:

Definition 2.3. A groupoid $\mathcal{G}$ consists of the following data:

- a set $\mathcal{G}_{1}$ of arrows (or morphisms),

- a set $\mathcal{G}_{0}$ of objects,

- a map $s: \mathcal{G}_{1} \rightarrow \mathcal{G}_{0}$ called the source map,

- a map $t: \mathcal{G}_{1} \rightarrow \mathcal{G}_{0}$ called the target map,

- a map

$$
m: \mathcal{G}_{1}{ }_{s} \times{ }_{t} \mathcal{G}_{1} \rightarrow \mathcal{G}_{0}, \quad(g, h) \mapsto m(g, h):=g h
$$

called the multiplication map, where

$$
\mathcal{G}_{1}{ }_{s} \times{ }_{t} \mathcal{G}_{1}:=\left\{(g, h) \in \mathcal{G}_{1} \times \mathcal{G}_{1} \mid s(g)=t(h)\right\},
$$

- a map $u: \mathcal{G}_{0} \rightarrow \mathcal{G}_{1}, x \mapsto u(x):=1_{x}$ called the unit map,

- $a$ map $\iota: \mathcal{G}_{1} \rightarrow \mathcal{G}_{1}, g \mapsto \iota(g):=g^{-1}$ called the inverse map

which satisfies the following conditions:

(i) $(g h) k=g(h k)$ for all $g, h, k \in \mathcal{G}_{1}$ satisfying $s(g)=t(h), s(h)=t(k)$,

(ii) $g 1_{s(g)}=1_{t(g)} g=g$ for all $g \in \mathcal{G}_{1}$,

(iii) $g^{-1} g=1_{s(g)}, g g^{-1}=1_{t(g)}$ for all $g \in \mathcal{G}_{1}$.

With regard to notation, one typically suppresses any mention of the structure maps and denotes the groupoid simply as

$$
\mathcal{G}_{1} \rightrightarrows \mathcal{G}_{0},
$$

or as the pair $\left(\mathcal{G}_{1}, \mathcal{G}_{0}\right)$. The groupoid is said to be finite if both $\mathcal{G}_{1}$ and $\mathcal{G}_{0}$ are finite.

REMARK 2.4. A groupoid $\mathcal{G}_{1} \rightrightarrows \mathcal{G}_{0}$ where $\mathcal{G}_{0}$ consists of a single point is simply a group. This is the motivation behind the name groupoid.

REMARK 2.5. The reader who is familiar with the notion of a category will instantly recognize that a groupoid is simply a small category where every morphism is invertible. This yields the shortest definition of a groupoid.

Now let $(M, G)$ be a finite global quotient and denote the left action of $G$ on $M$ by

$$
G \times M \rightarrow M, \quad(g, m) \mapsto g m .
$$

The groupoid of interest in this paper is the action groupoid $G \ltimes M$, which encodes all the information about $(M, G)$. Formally, the action groupoid is defined as follows:

1. the set of objects is $(G \ltimes M)_{0}:=M$,

2. the set of arrows is $(G \ltimes M)_{1}:=G \times M$, 
3. the source and target maps are $s(g, m):=m$ and $t(g, m):=g m$ for $g \in G, m \in M$,

4. composition of arrows is $(g, h m)(h, m):=(g h, m)$ for $g, h \in G, m \in M$,

5. the unit arrow associated to $m \in M$ is $1_{m}:=(e, m)$.

From the groupoid $G \ltimes M$, we obtain the groupoid algebra $\mathbb{k}[G \ltimes M]$, which has a natural weak Hopf structure. The weak Hopf algebra whose differential calculi we will study is then the dual of this groupoid algebra: $\mathbb{k}(G \ltimes M) \simeq$ $\mathbb{k}[G \ltimes M]^{*}$. As a vector space, the weak Hopf algebra $\mathbb{k}(G \ltimes M)$ consists of the $\mathbb{k}$-valued functions on the set $G \times M$. Let $\left\{\theta_{g}^{m} \mid(g, m) \in G \times M\right\}$ denote the natural basis on $\mathbb{k}(G \ltimes M)$, that is, for $(g, m) \in G \times M, \theta_{g}^{m}: G \times M \rightarrow \mathbb{k}$ is defined by

$$
\theta_{g}^{m}\left(g^{\prime}, m^{\prime}\right):=\delta_{g, g^{\prime}} \delta_{m, m^{\prime}}
$$

With respect to the natural basis, the weak Hopf structure on $\mathbb{k}(G \ltimes M)$ is defined by

1. product: $\theta_{g_{1}}^{m_{1}} \theta_{g_{2}}^{m_{2}}:=\delta_{g_{1}, g_{2}} \delta_{m_{1}, m_{2}} \theta_{g_{1}}^{m_{1}}$,

2. unit: $1:=\sum_{(g, m) \in G \times M} \theta_{g}^{m}$,

3. coproduct:

$$
\Delta\left(\theta_{g}^{m}\right):=\sum_{h \in G} \theta_{g h^{-1}}^{h m} \otimes \theta_{h}^{m},
$$

4. counit: $\varepsilon\left(\theta_{g}^{m}\right):=\delta_{g, e}$,

5. antipode: $S\left(\theta_{g}^{m}\right):=\theta_{g^{-1}}^{g m}$.

REMARK 2.6. Observe that $\mathbb{k}(G \ltimes M)$ fails to be a Hopf algebra when $M$ consists of more than one point. Indeed, if $m_{1}, m_{2} \in M$ are distinct points, then the counit fails to be multiplicative:

$$
\varepsilon\left(\theta_{e}^{m_{1}} \theta_{e}^{m_{2}}\right) \neq \varepsilon\left(\theta_{e}^{m_{1}}\right) \varepsilon\left(\theta_{e}^{m_{2}}\right) .
$$

The left side of (2.1) is

$$
\varepsilon\left(\theta_{e}^{m_{1}} \theta_{e}^{m_{2}}\right)=\delta_{m_{1}, m_{2}} \varepsilon\left(\theta_{e}^{m_{1}}\right)=\delta_{m_{1}, m_{2}}=0
$$

while the right side is

$$
\varepsilon\left(\theta_{e}^{m_{1}}\right) \varepsilon\left(\theta_{e}^{m_{2}}\right)=1
$$

\subsection{First Order Differential Calcui.}

DEFINITION 2.7. A first order differential calculus (FODC) over an algebra $A$ is a pair $(\Gamma, d)$ where $\Gamma$ is an $A$-bimodule and $d: A \rightarrow \Gamma$ is a linear map such that

(i) $\Gamma=\operatorname{span}\{a d b \mid a, b \in A\}$,

(ii) $d(a b)=a d b+(d a) b$.

The elements of $\Gamma$ are called 1-forms.

REMARK 2.8. Note that if $(\Gamma, d)$ is a FODC over $A$, then, in general, 1 -forms do not commute with elements of $A$ even if $A$ is commutative. 
Naturally, the above definition includes the following classical example.

EXAMPLE 2.9. Let $M$ be a compact manifold. Then $\left(\Omega^{1}(M), d\right)$ is a FODC over $C^{\infty}(M)$, where $\Omega^{1}(M)$ is the space of differential 1-forms on $M$ and $d: C^{\infty}(M) \rightarrow \Omega^{1}(M)$ is the ordinary exterior derivative. The condition of compactness implies that every 1 -form on $M$ can be expressed globally as $\sum_{k} f_{k} d g_{k}$ for some smooth functions $f_{k}, g_{k}$ on $M$.

Definition 2.10. Let $(\Gamma, d)$ and $\left(\Gamma^{\prime}, d^{\prime}\right)$ be FODCs over an algebra $A$. A morphism from $(\Gamma, d)$ to $\left(\Gamma^{\prime}, d^{\prime}\right)$ is a homomorphism of A-bimodules $\varphi: \Gamma \rightarrow$ $\Gamma^{\prime}$ such that $\varphi \circ d=d^{\prime}$. If $\varphi$ is also invertible, then $(\Gamma, d)$ and $\left(\Gamma^{\prime}, d^{\prime}\right)$ are isomorphic as FODCs.

Corollary 2.11. Let $(\Gamma, d)$ and $\left(\Gamma^{\prime}, d^{\prime}\right)$ be FODCs over an algebra $A$. If there exists a morphism $\varphi$ of FODCs over A from $(\Gamma, d)$ to $\left(\Gamma^{\prime}, d^{\prime}\right)$, then $\varphi$ is necessarily unique and surjective.

Proof. Suppose $\varphi:(\Gamma, d) \rightarrow\left(\Gamma^{\prime}, d^{\prime}\right)$ is a morphism of FODCs over $A$. Corollary 2.11 follows directly from the fact that

$$
\Gamma=\operatorname{span}\{a d b \mid a, b \in A\}, \quad \Gamma^{\prime}=\operatorname{span}\left\{a d^{\prime} b \mid a, b \in A\right\}
$$

and $\varphi(a d b)=a d^{\prime} b$ for all $a, b \in A$.

Every algebra $A$ admits a universal FODC $\left(\Omega_{u}^{1}(A), d_{u}\right)$, which is defined as follows. Let $\mu: A \otimes A \rightarrow A$ denote the product on $A$. Then

$$
\Omega_{u}^{1}(A):=\operatorname{ker} \mu
$$

and $d_{u}: A \rightarrow \Omega_{u}^{1}(A)$ is the linear map defined by

$$
d_{u}(a):=1 \otimes a-a \otimes 1 \quad \forall a \in A .
$$

The $A$-bimodule structure on $\Omega_{u}^{1}(A) \subset A \otimes A$ is the natural one, that is, for $\omega=\sum_{i} a_{i} \otimes b_{i} \in \Omega_{u}^{1}(A)$ and $c \in A$, the left and right action of $A$ on $\Omega_{u}^{1}(A)$ is given by

$$
c \omega=\sum_{i} c a_{i} \otimes b_{i}, \quad \omega c=\sum_{i} a_{i} \otimes b_{i} c .
$$

One can verify by direct calculation that $\left(\Omega_{u}^{1}(A), d_{u}\right)$ is a FODC over $A$. The use of the word universal is justified by the following result $([23,20])$ :

TheOREM 2.12. Let $A$ be an algebra and let $\left(\Omega_{u}^{1}(A), d_{u}\right)$ be the universal FODC over $A$.

(i) Let $W$ be an A-subbimodule of $\Omega_{u}^{1}(A)$. Then

$$
\left(\Omega_{u}^{1}(A) / W, \pi_{W} \circ d_{u}\right)
$$

is a FODC over $A$, where $\pi_{W}: \Omega_{u}^{1}(A) \rightarrow \Omega_{u}^{1}(A) / W$ is the projection map. 
(ii) Let $(\Gamma, d)$ be a FODC over $A$. Then there exists a homomorphism of FODCs

$$
\psi_{\Gamma}:\left(\Omega_{u}^{1}(A), d_{u}\right) \rightarrow(\Gamma, d) .
$$

In particular, for $W=\operatorname{ker} \psi_{\Gamma}$, the induced map

$$
\widetilde{\psi}_{\Gamma}:\left(\Omega_{u}^{1}(A) / W, \pi_{W} \circ d_{u}\right) \stackrel{\sim}{\rightarrow}(\Gamma, d)
$$

is an isomorphism of FODCs.

If $A$ is now a Hopf algebra, one typically restricts attention to FODCs which are left-covariant, right-covariant, or bicovariant ([23]). The aforementioned symmetries extend naturally to FODCs over weak Hopf algebras:

Definition 2.13. Let $(\Gamma, d)$ be a FODC over a weak Hopf algebra $H$.

1. $(\Gamma, d)$ is left-covariant if there exists a left $H$-coaction

$$
\Delta_{L}: \Gamma \rightarrow H \otimes \Gamma
$$

such that

2. $(\Gamma, d)$ is right-covariant if there exists a right $H$-coaction

$$
\Delta_{R}: \Gamma \rightarrow \Gamma \otimes H
$$

such that

$$
\Delta_{R}(a d b)=a_{(1)} d b_{(1)} \otimes a_{(2)} b_{(2)}, \quad \forall a, b \in H .
$$

3. $(\Gamma, d)$ is bicovariant if it is both left and right-covariant.

REMARK 2.14. Note that if $(\Gamma, d)$ is a bicovariant FODC over a weak Hopf algebra $H$, then the covariant left and right coactions necessarily form a bicomodule structure on $\Gamma$, that is,

$$
\left(i d \otimes \Delta_{R}\right) \circ \Delta_{L}=\left(\Delta_{L} \otimes i d\right) \circ \Delta_{R} .
$$

Suppose now that one has linear maps

$$
\delta_{L}: \Gamma \rightarrow H \otimes \Gamma, \quad \delta_{R}: \Gamma \rightarrow \Gamma \otimes H
$$

which satisfy the covariance conditions (2.4) and (2.5) respectively. When $H$ is a Hopf algebra, $\delta_{L}$ and $\delta_{R}$ are easily seen to be coactions of $H$ on $\Gamma$. On the other hand, if $H$ is a weak Hopf algebra (which is not Hopf), then the covariance conditions (2.4) and (2.5) no longer seem to be sufficient to guarantee that $\delta_{L}$ and $\delta_{R}$ are coactions. The reason for this comes from the fact that the counit $\varepsilon$ is now only weakly multiplicative. Consequently, it is no longer clear that the following conditions necessarily hold:

$$
(\varepsilon \otimes i d) \circ \delta_{L}=i d, \quad(i d \otimes \varepsilon) \circ \delta_{R}=i d .
$$

Hence, in the weak Hopf case, one must take the additional step of verifying the coaction axioms directly. 
One additional difference between the Hopf and weak Hopf cases concerns the universal FODC. When $H$ is a Hopf algebra, the universal FODC over $H$ is necessarily bicovariant (see e.g. [20]). On the other hand, when $H$ is a weak Hopf algebra, the universal FODC over $H$ is not necessarily bicovariant. We will observe this feature later when we study bicovariant FODCs over the weak Hopf algebra $\mathbb{k}(G \ltimes M)$.

\section{Review of Differential Calculi on Finite Sets}

In this section, we give a brief review of FODC on finite sets $([9,8])$. Throughout this section, $X$ is a finite set, $\mathbb{k}(X)$ is the algebra of $\mathbb{k}$-valued functions on $X$, and for $x \in X$, we let $\delta_{x}: X \rightarrow \mathbb{k}$ be defined by $\delta_{x}(y):=\delta_{x, y}$ for all $y \in X$. In addition, we let $\left(\Omega_{u}^{1}(X), d_{u}\right)$ denote the universal FODC over $\mathbb{k}(X)$.

Proposition 3.1. Let $E_{u}:=\{(x, y) \mid x, y \in X, x \neq y\} \subset X \times X$. Then

(i) $\widehat{E}_{u}:=\left\{\delta_{x} d_{u} \delta_{y} \mid(x, y) \in E_{u}\right\}$ is a vector space basis of $\Omega_{u}^{1}(X)$.

(ii) Let $N \subset \Omega_{u}^{1}(X)$ be any vector subspace. Then $N$ is a $\mathbb{k}(X)$-subbimodule of $\Omega_{u}^{1}(X)$ if and only if there exists a unique subset $B_{N} \subset E_{u}$ such that

$$
\widehat{B}_{N}:=\left\{\delta_{x} d_{u} \delta_{y} \mid(x, y) \in B_{N}\right\}
$$

is a vector space basis of $N$.

(iii) $\delta_{x} d_{u} \delta_{x}=-\sum_{y \neq x} \delta_{x} \otimes \delta_{y}$ for $x \in X$.

Proof. (i): Since $\left\{\delta_{x} \mid x \in X\right\}$ is a basis of $\mathbb{k}(X)$ and

$$
\delta_{x} d_{u} \delta_{y}=\delta_{x} \otimes \delta_{y}-\delta_{x, y} \delta_{x} \otimes 1=\delta_{x} \otimes \delta_{y} \quad \text { for } x \neq y,
$$

it follows immediately that $\widehat{E}_{u}$ is a linearly independent subset of $\Omega_{u}^{1}(X) \subset$ $\mathbb{k}(X) \otimes \mathbb{k}(X)$. Also, since the product map $\mathbb{k}(X) \otimes \mathbb{k}(X) \rightarrow \mathbb{k}(X)$ is surjective, we have

$$
\operatorname{dim} \Omega_{u}^{1}(X)=|X|^{2}-|X|=|X|(|X|-1)
$$

where $|X|$ denotes the cardinality of $X$. However, the right hand side of (3.1) is also equal to the cardinality of $\widehat{E}_{u}$. Hence, $\widehat{E}_{u}$ is a vector space basis of $\Omega_{u}^{1}(X)$.

Then

(ii): Suppose that $N$ is a $\mathbb{k}(X)$-subbimodule of $\Omega_{u}^{1}(X)$ and let $\omega \in N$.

$$
\omega=\sum_{(x, y) \in E_{u}} \omega_{x, y} \delta_{x} d_{u} \delta_{y}=\sum_{(x, y) \in E_{u}} \omega_{x, y} \delta_{x} \otimes \delta_{y}
$$

for some (unique) $\omega_{x, y} \in \mathbb{k}$. Since $N$ is a subbimodule, we have

$$
\delta_{x} \omega \delta_{y}=\omega_{x, y} \delta_{x} \otimes \delta_{y} \in N
$$

for $(x, y) \in E_{u}$. This implies that $N$ is the $\mathbb{k}$-linear span of some subset $\widehat{B}_{N}$ of $\widehat{E}_{u}$. Since the elements of $\widehat{E}_{u}$ are linearly independent, $\widehat{B}_{N}$ is necessarily 
a vector space basis of $N$. The linear independence of $\widehat{E}_{u}$ also implies that $\widehat{B}_{N}$ is the only subset of $\widehat{E}_{u}$ which is a vector space basis for $N$. Since the elements of $\widehat{E}_{u}$ are indexed by the elements of $E_{u}$, there exists a unique subset $B_{N}$ of $E_{u}$ such that

$$
\widehat{B}_{N}=\left\{\delta_{x} d_{u} \delta_{y} \mid(x, y) \in B_{N}\right\} .
$$

Conversely, suppose $N$ is a vector subspace of $\Omega_{u}^{1}(X)$ such that there exists a subset $B_{N} \subset E_{u}$ for which $\left\{\delta_{x} d_{u} \delta_{y} \mid(x, y) \in B_{N}\right\}$ is a vector space basis of $N$. Let $f \in \mathbb{k}(X)$. Then for $(x, y) \in B_{N}$, we have

$$
f \cdot\left(\delta_{x} d_{u} \delta_{y}\right)=f(x) \delta_{x} d_{u} \delta_{y}, \quad\left(\delta_{x} d_{u} \delta_{y}\right) \cdot f=f(y) \delta_{x} d_{u} \delta_{y} .
$$

This implies that $N$ is a $\mathbb{k}(X)$-subbimodule of $\Omega_{u}^{1}(X)$.

(iii): For $x \in X$, we have

$$
\begin{aligned}
0 & =\delta_{x} d_{u} 1=\delta_{x} d_{u} \delta_{x}+\sum_{y \neq x} \delta_{x} d_{u} \delta_{y} \\
& =\delta_{x} d_{u} \delta_{x}+\sum_{y \neq x} \delta_{x}\left(1 \otimes \delta_{y}-\delta_{y} \otimes 1\right) \\
& =\delta_{x} d_{u} \delta_{x}+\sum_{y \neq x} \delta_{x} \otimes \delta_{y} .
\end{aligned}
$$

The last equality yields (iii).

Definition 3.2. For a FODC $(\Gamma, d)$ over $\mathbb{k}(X)$, let $B_{\Gamma}$ and $E_{\Gamma}$ be the unique subsets of $E_{u}$ defined by

$$
B_{\Gamma}:=\left\{(x, y) \in E_{u} \mid \delta_{x} d \delta_{y}=0\right\}, \quad E_{\Gamma}:=E_{u}-B_{\Gamma} .
$$

Proposition 3.3. Let $(\Gamma, d)$ and $\left(\Gamma^{\prime}, d^{\prime}\right)$ be FODCs over $\mathbb{k}(X)$. Then

(i) $\widehat{E}_{\Gamma}:=\left\{\delta_{x} d \delta_{y} \mid(x, y) \in E_{\Gamma}\right\}$ is a vector space basis of $\Gamma$

(ii) $(\Gamma, d)$ and $\left(\Gamma^{\prime}, d^{\prime}\right)$ are isomorphic as FODCs if and only if $E_{\Gamma}=E_{\Gamma^{\prime}}$

Proof. (i): By Theorem 2.12, there exists a homomorphism of FODCs

$$
\psi_{\Gamma}:\left(\Omega_{u}^{1}(X), d_{u}\right) \rightarrow(\Gamma, d) .
$$

By Corollary 2.11, $\psi_{\Gamma}$ is necessarily a (unique) surjective homomorphism of $\mathbb{k}(X)$-bimodules. Since $\mathrm{ker} \psi_{\Gamma}$ is a $\mathbb{k}(X)$-subbimodule of $\Omega_{u}^{1}(X)$, Proposition 3.1 implies that there exists a unique subset $K \subset E_{u}$ such that $\widehat{K}:=\left\{\delta_{x} d_{u} \delta_{y} \mid(x, y) \in K\right\}$ is a basis of $\operatorname{ker} \psi_{\Gamma}$. For $(x, y) \in K$, we have $\psi_{\Gamma}\left(\delta_{x} d_{u} \delta_{y}\right)=\delta_{x} d \delta_{y}=0$ which shows that $K \subset B_{\Gamma}$. On the other hand, by definition of $B_{\Gamma}$, if $\left(x^{\prime}, y^{\prime}\right) \in B_{\Gamma}$, then $\delta_{x^{\prime}} d \delta_{y^{\prime}}=0$. This in turn implies that $\delta_{x^{\prime}} d_{u} \delta_{y^{\prime}} \in \operatorname{ker} \psi_{\Gamma}$. Since the elements of $\widehat{E}_{u}$ are a vector space basis on $\Omega_{u}^{1}(X)$ by Proposition 3.1, it follows that $\delta_{x^{\prime}} d_{u} \delta_{y^{\prime}} \in \widehat{K}$, which in turn implies that 
$\left(x^{\prime}, y^{\prime}\right) \in K$. This shows that $B_{\Gamma}=K$. This implies that as a vector space, $\Gamma$ is spanned by the elements

$$
\widehat{E}_{\Gamma}:=\left\{\delta_{x} d \delta_{y} \mid(x, y) \in E_{\Gamma}:=E_{u}-B_{\Gamma}\right\} .
$$

However, the vector space dimension of $\Gamma$ is

$$
\operatorname{dim} \Gamma=\operatorname{dim} \Omega_{u}^{1}(X)-\operatorname{dim} \operatorname{ker} \psi_{\Gamma}=\left|E_{u}-B_{\Gamma}\right|=\left|E_{\Gamma}\right|
$$

where $|\cdot|$ denotes the cardinality of a set. Hence, $\widehat{E}_{\Gamma}$ is a vector space basis of $\Gamma$.

(ii): Suppose that $(\Gamma, d)$ and $\left(\Gamma^{\prime}, d^{\prime}\right)$ are isomorphic as FODCs and let $\varphi:(\Gamma, d) \stackrel{\sim}{\rightarrow}\left(\Gamma^{\prime}, d^{\prime}\right)$ be an isomorphism of FODCs over $\mathbb{k}(X)$. (Note that $\varphi$ is unique by Corollary 2.11.) For $(x, y) \in B_{\Gamma}$, we have

$$
0=\varphi(0)=\varphi\left(\delta_{x} d \delta_{y}\right)=\delta_{x} d^{\prime} \delta_{y},
$$

which shows that $B_{\Gamma} \subset B_{\Gamma^{\prime}}$. Using the inverse map $\varphi^{-1}$, one has $B_{\Gamma} \supset B_{\Gamma^{\prime}}$. Hence, $B_{\Gamma}=B_{\Gamma^{\prime}}$ which in turn implies that $E_{\Gamma}=E_{\Gamma^{\prime}}$.

Now suppose that $E_{\Gamma}=E_{\Gamma^{\prime}}$. This in turn implies that $B_{\Gamma}=B_{\Gamma^{\prime}}$. Let

$$
\psi_{\Gamma}:\left(\Omega_{u}^{1}(X), d_{u}\right) \rightarrow(\Gamma, d), \quad \psi_{\Gamma^{\prime}}:\left(\Omega_{u}^{1}(X), d_{u}\right) \rightarrow\left(\Gamma^{\prime}, d^{\prime}\right)
$$

be the homomorphisms of FODCs over $\mathbb{k}(X)$ given by Theorem 2.12. (By Corollary 2.11, $\psi_{\Gamma}$ and $\psi_{\Gamma^{\prime}}$ are unique and surjective.) From the proof of statement (i) of Proposition 3.3, we see that

$$
\left\{\delta_{x} d_{u} \delta_{y} \mid(x, y) \in B_{\Gamma}=B_{\Gamma^{\prime}}\right\}
$$

is a vector space basis of both $\operatorname{ker} \psi_{\Gamma}$ and $\operatorname{ker} \psi_{\Gamma^{\prime}}$. Hence, $\operatorname{ker} \psi_{\Gamma}=\operatorname{ker} \psi_{\Gamma^{\prime}}$. Let $W:=\operatorname{ker} \psi_{\Gamma}$. By Theorem 2.12, we have the following isomorphisms of FODCs

$$
(\Gamma, d) \simeq\left(\Omega_{u}^{1}(X) / W, \pi_{W} \circ d_{u}\right) \simeq\left(\Gamma^{\prime}, d^{\prime}\right)
$$

where $\pi_{W}: \Omega_{u}^{1}(X) \rightarrow \Omega_{u}^{1}(X) / W$ is the natural projection.

We conclude this section by recalling the following correspondence $([9,8])$.

THEOREM 3.4. There is a one-to-one correspondence between isomorphism classes of FODCs over $\mathbb{k}(X)$ and directed graphs whose vertices are the elements of $X$ and have the property that for each $(x, y) \in E_{u}$ there is at most one arrow from $x$ to $y$.

Proof. Let $(\Gamma, d)$ be a FODC over $\mathbb{k}(X)$. Let $G_{\Gamma}$ be the directed graph whose vertices are the elements of $X$ and has the property that there is exactly one directed edge from $x \in X$ to $y \in X$ if and only if $(x, y) \in E_{\Gamma}$. Proposition 3.3 implies that if $\left(\Gamma^{\prime}, d^{\prime}\right)$ is another FODC over $\mathbb{k}(X)$, then $G_{\Gamma}=G_{\Gamma^{\prime}}$ if and only if $(\Gamma, d)$ and $\left(\Gamma^{\prime}, d^{\prime}\right)$ are isomorphic. Hence, every isomorphism class of FODCs over $\mathbb{k}(X)$ is uniquely associated to some directed graph whose vertices are the elements of $X$ and has the property that for each $(x, y) \in E_{u}$ there is at most one directed edge from $x$ to $y$. 
Now suppose one has a directed graph $\mathcal{G}$ whose vertices are the elements of $X$ and has the property that for each $(x, y) \in E_{u}$ there is at most one directed edge from $x$ to $y$. We now associate a FODC over $\mathbb{k}(X)$ to $\mathcal{G}$, which we denote as $\left(\Gamma_{\mathcal{G}}, d_{\mathcal{G}}\right)$. First, let $E_{\mathcal{G}} \subset E_{u}$ be the set of all pairs $(x, y)$ such that $\mathcal{G}$ has a directed edge from $x$ to $y$. Let $B_{\mathcal{G}}=E_{u}-E_{\mathcal{G}}$ be the complement. Let $W$ be the vector subspace of $\Omega_{u}^{1}(X)$ with vector space basis

$$
\left\{\delta_{x} d_{u} \delta_{y} \mid(x, y) \in B_{\mathcal{G}}\right\} .
$$

By Proposition 3.1, $W$ is a subbimodule of $\Omega_{u}^{1}(X)$. Let $\Gamma_{\mathcal{G}}:=\Omega_{u}^{1}(X) / W$ and $d_{\mathcal{G}}:=\pi_{W} \circ d_{u}$ where $\pi_{W}: \Omega_{u}^{1}(X) \rightarrow \Omega_{u}^{1}(X) / W$ is the natural projection. Then $\left(\Gamma_{\mathcal{G}}, d_{\mathcal{G}}\right)$ is a FODC over $\mathbb{k}(X)$ by Theorem 2.12.

We now verify that the above constructions are inverse to one another. Suppose first that $\mathcal{G}$ is a directed graph whose vertices are the elements of $X$ and has the property that for each $(x, y) \in E_{u}$ there is at most one directed edge from $x$ to $y$. Using the above definition of $\Gamma_{\mathcal{G}}$, we have

$$
B_{\Gamma_{\mathcal{G}}}:=\left\{(x, y) \in E_{u} \mid \delta_{x} d_{\mathcal{G}} \delta_{y}=0\right\}=B_{\mathcal{G}} .
$$

Hence,

$$
E_{\Gamma_{\mathcal{G}}}:=E_{u}-B_{\Gamma_{\mathcal{G}}}=E_{u}-B_{\mathcal{G}}=E_{\mathcal{G}},
$$

which implies that $G_{\Gamma_{\mathcal{G}}}=\mathcal{G}$ as required.

On the other hand, suppose that $(\Gamma, d)$ is a FODC over $\mathbb{k}(X)$. From the above definitions, we have

$$
E_{\Gamma}:=\left\{(x, y) \in E_{u} \mid \delta_{x} d \delta_{y} \neq 0\right\}=E_{G_{\Gamma}} .
$$

Consequently, as a vector space, $\Gamma_{G_{\Gamma}}$ has basis

$$
\left\{\delta_{x} d_{G_{\Gamma}} \delta_{y} \mid(x, y) \in E_{G_{\Gamma}}=E_{\Gamma}\right\} .
$$

This implies that the homomorphisms

$$
\psi_{\Gamma}: \Omega_{u}^{1}(X) \rightarrow \Gamma, \quad \psi_{\Gamma_{\mathcal{G}}}: \Omega_{u}^{1}(X) \rightarrow \Gamma_{G_{\Gamma}}
$$

have the same kernels. It follows from this that $(\Gamma, d) \simeq\left(\Gamma_{G_{\Gamma}}, d_{G_{\Gamma}}\right)$ as required.

\section{Bicovariant FODC over $\mathbb{k}(G \ltimes M)$}

In this section, we let $\left(\Omega_{u}^{1}, d_{u}\right)$ denote the universal FODC over $\mathbb{k}(G \ltimes M)$. Also, let

$$
\left\{\theta_{g}^{m} \mid m \in M, g \in G\right\}
$$

denote the natural basis on $\mathbb{k}(G \ltimes M)$ (see Section 2.2). Following Section 3, we define

$$
E_{u}:=\{((g, m),(h, n)) \mid(g, m) \neq(h, n), g, h \in G, m, n \in M\} .
$$


By Theorem 3.4, there is a one-to-one correspondence between isomorphism classes of FODC over $\mathbb{k}(G \ltimes M)$ and subsets of $E_{u}$. Explicitly, the FODC associated to a subset $E \subset E_{u}$ is given by

$$
\left(\Omega_{u}^{1} / W, \pi_{W} \circ d_{u}\right)
$$

where

$$
W:=\operatorname{span}_{\mathbb{k}}\left\{\theta_{g}^{m} d_{u} \theta_{h}^{n} \mid((g, m),(h, n)) \in E_{u}-E\right\}
$$

and $\pi_{W}: \Omega_{u}^{1} \rightarrow \Omega_{u}^{1} / W$ is the natural projection. (Recall from Proposition 3.1 that $W$ is a $\mathbb{k}(G \ltimes M)$-subbimodule of $\Omega_{u}^{1}$ and all subbimodules of $\Omega_{u}^{1}$ are of this form.) From Section 3, the subset of $E_{u}$ which is associated to a FODC $(\Gamma, d)$ over $\mathbb{k}(G \ltimes M)$ is

$$
E_{\Gamma}:=\left\{((g, m),(h, n)) \mid \theta_{g}^{m} d \theta_{h}^{n} \neq 0,((g, m),(h, n)) \in E_{u}\right\} .
$$

Following Section 3 , we also define $B_{\Gamma}:=E_{u}-E_{\Gamma}$. For $m \in M$, let $G_{m}:=$ $\{g \in G \mid g m=m\}$ denote the isotropy group of $m$. Let us now introduce the following definition.

Definition 4.1. A subset $B$ of $E_{u}$ is bicovariant if the following three conditions are satisfied:

(1) for $m, n \in M$ such that $m \neq n,((g, m),(h, n)) \in B$ for all $g, h \in G$,

(2) for $g, h \in G, m \in M$ such that $g^{-1} h \notin G_{m},((g, m),(h, m)) \in B$,

(3) if $((g, m),(g k, m)) \in B$ for some $g \in G, m \in M$, and $k \in G_{m}-\{e\}$, then

$$
\left((h, y m),\left(h y k y^{-1}, y m\right)\right) \in B \quad \forall h, y \in G .
$$

The justification for Definition 4.1 comes from the following result.

TheOREm 4.2. Let $(\Gamma, d)$ be a FODC over $\mathbb{k}(G \ltimes M)$. Then $(\Gamma, d)$ is bicovariant if and only if $B_{\Gamma}$ is bicovariant in the sense of Definition 4.1.

Theorem 4.2 can be restated as follows.

Corollary 4.3. Let $(\Gamma, d)$ be a FODC over $\mathbb{k}(G \ltimes M)$. Then $(\Gamma, d)$ is bicovariant if and only if the following three conditions are satisfied:

(i) for $m, n \in M$ such that $m \neq n, \theta_{g}^{m} d \theta_{h}^{n}=0$ for all $g, h \in G$,

(ii) for $g, h \in G, m \in M$ such that $g^{-1} h \notin G_{m}, \theta_{g}^{m} d \theta_{h}^{m}=0$,

(iii) if $\theta_{g}^{m} d \theta_{g k}^{m}=0$ for some $g \in G, m \in M$, and $k \in G_{m}-\{e\}$, then

$$
\theta_{h}^{y m} d \theta_{h y k y^{-1}}^{y m}=0 \quad \forall h, y \in G .
$$

We now turn our attention to proving Theorem 4.2. We begin with the following lemma.

Lemma 4.4. Let $(\Gamma, d)$ be a FODC over $\mathbb{k}(G \ltimes M)$ such that $B_{\Gamma}$ satisfies conditions (1) and (2) of Definition 4.1. If

$$
\delta_{L}: \Gamma \rightarrow \mathbb{k}(G \ltimes M) \otimes \Gamma, \quad \delta_{R}: \Gamma \rightarrow \Gamma \otimes \mathbb{k}(G \ltimes M),
$$


are linear maps satisfying

$$
\begin{aligned}
& \delta_{L}\left(\theta_{g}^{m} d \theta_{h}^{n}\right)=\left(\theta_{g}^{m}\right)_{(1)}\left(\theta_{h}^{n}\right)_{(1)} \otimes\left(\theta_{g}^{m}\right)_{(2)} d\left(\theta_{h}^{n}\right)_{(2)}, \\
& \delta_{R}\left(\theta_{g}^{m} d \theta_{h}^{n}\right)=\left(\theta_{g}^{m}\right)_{(1)} d\left(\theta_{h}^{n}\right)_{(1)} \otimes\left(\theta_{g}^{m}\right)_{(2)}\left(\theta_{h}^{n}\right)_{(2)}
\end{aligned}
$$

for all $\theta_{g}^{m} \neq \theta_{h}^{n}$, then $\delta_{L}$ and $\delta_{R}$ satisfy the covariance conditions (2.4) and (2.5).

Proof. Since $\left\{\theta_{g}^{m} \mid m \in M, g \in G\right\}$ is a basis on $\mathbb{k}(G \ltimes M)$, it suffices to check that $\delta_{L}$ and $\delta_{R}$ satisfy the covariance conditions (2.4) and (2.5) on elements of the form $\theta_{g}^{m} d \theta_{g}^{m}$.

Since the unit element of $\mathbb{k}(G \ltimes M)$ is $1=\sum_{n \in M} \sum_{h \in G} \theta_{h}^{n}$ and $d 1=0$, it follows that

$$
\theta_{g}^{m} d \theta_{g}^{m}=-\sum_{\left\{\theta_{h}^{n} \mid \theta_{h}^{n} \neq \theta_{g}^{m}\right\}} \theta_{g}^{m} d \theta_{h}^{n}=-\sum_{k \in G_{m}-\{e\}} \theta_{g}^{m} d \theta_{g k}^{m},
$$

where the last equality follows from the fact that $B_{\Gamma}$ satisfies conditions (1) and (2) of Definition 4.1 (which in turn are equivalent to conditions (i) and (ii) of Corollary 4.3). Applying $\delta_{L}$ to both sides of (4.3) and using (4.1) gives

$$
\begin{aligned}
\delta_{L}\left(\theta_{g}^{m} d \theta_{g}^{m}\right) & =-\sum_{k \in G_{m}-\{e\}}\left(\theta_{g}^{m}\right)_{(1)}\left(\theta_{g k}^{m}\right)_{(1)} \otimes\left(\theta_{g}^{m}\right)_{(2)} d\left(\theta_{g k}^{m}\right)_{(2)} \\
& =-\sum_{k \in G_{m}-\{e\}} \sum_{x, y \in G} \theta_{g x^{-1}}^{x m} \theta_{g k y^{-1}}^{y m} \otimes \theta_{x}^{m} d \theta_{y}^{m} \\
& =-\sum_{k \in G_{m}-\{e\}} \sum_{x \in G} \theta_{g x^{-1}}^{x m} \otimes \theta_{x}^{m} d \theta_{x k}^{m} \\
& =\sum_{x \in G}\left[-\sum_{k \in G_{m}-\{e\}} \theta_{g x^{-1}}^{x m} \otimes \theta_{x}^{m} d \theta_{x k}^{m}\right] \\
& =\sum_{x \in G} \theta_{g x^{-1}}^{x m} \otimes \theta_{x}^{m} d \theta_{x}^{m},
\end{aligned}
$$

where the last equality follows from (4.3). On the other hand,

$$
\begin{aligned}
\left(\theta_{g}^{m}\right)_{(1)}\left(\theta_{g}^{m}\right)_{\left(1^{\prime}\right)} \otimes\left(\theta_{g}^{m}\right)_{(2)} d\left(\theta_{g}^{m}\right)_{\left(2^{\prime}\right)} & =\sum_{x, y \in G} \theta_{g x^{-1}}^{x m} \theta_{g y^{-1}}^{y m} \otimes \theta_{x}^{m} d \theta_{y}^{m} \\
& =\sum_{x \in G} \theta_{g x^{-1}}^{x m} \otimes \theta_{x}^{m} d \theta_{x}^{m} .
\end{aligned}
$$

Comparing (4.4) and (4.5) gives

$$
\delta_{L}\left(\theta_{g}^{m} d \theta_{g}^{m}\right)=\left(\theta_{g}^{m}\right)_{(1)}\left(\theta_{g}^{m}\right)_{\left(1^{\prime}\right)} \otimes\left(\theta_{g}^{m}\right)_{(2)} d\left(\theta_{g}^{m}\right)_{\left(2^{\prime}\right)} .
$$


BICOVARIANT DIFFERENTIAL CALCULI FOR FINITE GLOBAL QUOTIENTS 491

Applying $\delta_{R}$ to both sides of (4.3) and using (4.2) gives

$$
\begin{aligned}
\delta_{R}\left(\theta_{g}^{m} d \theta_{g}^{m}\right) & =-\sum_{k \in G_{m}-\{e\}}\left(\theta_{g}^{m}\right)_{(1)} d\left(\theta_{g k}^{m}\right)_{(1)} \otimes\left(\theta_{g}^{m}\right)_{(2)}\left(\theta_{g k}^{m}\right)_{(2)} \\
& =-\sum_{k \in G_{m}-\{e\}} \sum_{x, y \in G} \theta_{g x^{-1}}^{x m} d \theta_{g k y^{-1}}^{y m} \otimes \theta_{x}^{m} \theta_{y}^{m} \\
& =\sum_{x \in G}\left[-\sum_{k \in G_{m}-\{e\}} \theta_{g x^{-1}}^{x m} d \theta_{g k x^{-1}}^{x m} \otimes \theta_{x}^{m}\right] \\
& =\sum_{x \in G}\left[-\sum_{k \in G_{m}-\{e\}} \theta_{g x^{-1}}^{x m} d \theta_{g x^{-1}\left(x k x^{-1}\right)}^{x m} \otimes \theta_{x}^{m}\right] \\
& =\sum_{x \in G}\left[-\sum_{k^{\prime} \in G_{x m}-\{e\}} \theta_{g x^{-1}}^{x m} d \theta_{g x^{-1} k^{\prime}}^{x m} \otimes \theta_{x}^{m}\right] \\
& =\sum_{x \in G} \theta_{g x^{-1}}^{x m} d \theta_{g x^{-1}}^{x m} \otimes \theta_{x}^{m},
\end{aligned}
$$

where the last equality follows from (4.3). At the same time, we also have

$$
\begin{aligned}
\left(\theta_{g}^{m}\right)_{(1)} d\left(\theta_{g}^{m}\right)_{\left(1^{\prime}\right)} \otimes\left(\theta_{g}^{m}\right)_{(2)}\left(\theta_{g}^{m}\right)_{\left(2^{\prime}\right)} & =\sum_{x, y \in G} \theta_{g x^{-1}}^{x m} d \theta_{g y^{-1}}^{y m} \otimes \theta_{x}^{m} \theta_{y}^{m} \\
& =\sum_{x \in G} \theta_{g x^{-1}}^{x m} d \theta_{g x^{-1}}^{x m} \otimes \theta_{x}^{m} .
\end{aligned}
$$

Comparing (4.6) and (4.7) gives

$$
\delta_{R}\left(\theta_{g}^{m} d \theta_{g}^{m}\right)=\left(\theta_{g}^{m}\right)_{(1)} d\left(\theta_{g}^{m}\right)_{\left(1^{\prime}\right)} \otimes\left(\theta_{g}^{m}\right)_{(2)}\left(\theta_{g}^{m}\right)_{\left(2^{\prime}\right)} .
$$

Proposition 4.5. Let $(\Gamma, d)$ be a bicovariant FODC over $\mathbb{k}(G \ltimes M)$. Then $B_{\Gamma}$ is bicovariant in the sense of Definition 4.1.

Proof. Let $\Delta_{L}$ and $\Delta_{R}$ denote the left and right-covariant coactions respectively. Applying $\Delta_{R}$ and $\Delta_{L}$ to $\theta_{g}^{m} d \theta_{h}^{n}$ gives

$$
\begin{aligned}
\Delta_{R}\left(\theta_{g}^{m} d \theta_{h}^{n}\right) & =\sum_{x, y \in G} \theta_{g x^{-1}}^{x m} d \theta_{h y^{-1}}^{y n} \otimes \theta_{x}^{m} \theta_{y}^{n} \\
& =\sum_{x, y \in G} \delta_{m, n} \delta_{x, y} \theta_{g x^{-1}}^{x m} d \theta_{h y^{-1}}^{y n} \otimes \theta_{x}^{m} \\
& =\sum_{x \in G} \delta_{m, n} \theta_{g x^{-1}}^{x m} d \theta_{h x^{-1}}^{x n} \otimes \theta_{x}^{m}
\end{aligned}
$$


and

$$
\begin{aligned}
\Delta_{L}\left(\theta_{g}^{m} d \theta_{h}^{n}\right) & =\sum_{x, y \in G} \theta_{g x^{-1}}^{x m} \theta_{h y^{-1}}^{y n} \otimes \theta_{x}^{m} d \theta_{y}^{n} \\
& =\sum_{x, y \in G} \delta_{x m, y n} \delta_{g x^{-1}, h y^{-1}} \theta_{g x^{-1}}^{x m} \otimes \theta_{x}^{m} d \theta_{y}^{n} .
\end{aligned}
$$

Since $(i d \otimes \varepsilon) \circ \Delta_{R}=i d$, equation (4.8) implies that

$$
\theta_{g}^{m} d \theta_{h}^{n}=\sum_{x \in G} \delta_{m, n} \delta_{x, e} \theta_{g x^{-1}}^{x m} d \theta_{h x^{-1}}^{x n}=\delta_{m, n} \theta_{g}^{m} d \theta_{h}^{n} .
$$

Hence, $\theta_{g}^{m} d \theta_{h}^{n}=0$ for $m \neq n$. This implies that condition (1) of Definition 4.1 is satisfied.

Likewise, since $(\varepsilon \otimes i d) \circ \Delta_{L}=i d$, equation (4.9) implies that

$$
\theta_{g}^{m} d \theta_{h}^{n}=\sum_{x, y \in G} \delta_{x m, y n} \delta_{g x^{-1}, h y^{-1}} \delta_{g x^{-1}, e} \theta_{x}^{m} d \theta_{y}^{n}=\delta_{g m, h n} \theta_{g}^{m} d \theta_{h}^{n} .
$$

From (4.10), we see that if $m=n$ and $g^{-1} h \notin G_{m}$, then

$$
\theta_{g}^{m} d \theta_{h}^{m}=\delta_{g m, h m} \theta_{g}^{m} d \theta_{h}^{m}=\delta_{m, g^{-1} h m} \theta_{g}^{m} d \theta_{h}^{m}=0 .
$$

Condition (2) of Definition 4.1 now follows from (4.11).

Now suppose that $\theta_{g}^{m} d \theta_{g k}^{m}=0$ for some $g \in G, m \in M$, and $k \in G_{m}-\{e\}$. Equations (4.8) and (4.9) then imply

$$
\sum_{x \in G} \theta_{g x^{-1}}^{x m} d \theta_{g k x^{-1}}^{x m} \otimes \theta_{x}^{m}=0
$$

and

$$
\sum_{x \in G} \theta_{g x^{-1}}^{x m} \otimes \theta_{x}^{m} d \theta_{x k}^{m}=0 .
$$

Since $\left\{\theta_{h}^{n} \mid n \in M, h \in G\right\}$ is a basis of $\mathbb{k}(G \ltimes M)$, equations (4.12) and (4.13) imply

$$
\theta_{g x^{-1}}^{x m} d \theta_{g k x^{-1}}^{x m}=0, \quad \theta_{x}^{m} d \theta_{x k}^{m}=0, \quad \forall x \in G .
$$

Using (4.14), we have

$$
\Delta_{R}\left(\theta_{x}^{m} d \theta_{x k}^{m}\right)=\sum_{a \in G} \theta_{x a^{-1}}^{a m} d \theta_{x k a^{-1}}^{a m} \otimes \theta_{a}^{m}=0, \quad \forall x \in G .
$$

This implies that $\theta_{x a^{-1}}^{a m} d \theta_{x k a^{-1}}^{a m}=0$ for all $x, a \in G$. Now let $h, y \in G$ be arbitrary and set $a:=y$ and $x:=h y$. Then

$$
\theta_{x a^{-1}}^{a m} d \theta_{x k a^{-1}}^{a m}=\theta_{(h y) y^{-1}}^{y m} d \theta_{(h y) y^{-1}\left(y k y^{-1}\right)}^{y m}=\theta_{h}^{y m} d \theta_{h y k y^{-1}}^{y m}=0 .
$$

This implies that condition (3) of Definition 4.1 is satisfied. 
BICOVARIANT DIFFERENTIAL CALCULI FOR FINITE GLOBAL QUOTIENTS 493

Lemma 4.6. Let $(\Gamma, d)$ be a FODC over $\mathbb{k}(G \ltimes M)$ such that $B_{\Gamma}$ is bicovariant in the sense of Definition 4.1. If $((g, m),(h, n)) \in B_{\Gamma}$, then $\theta_{g}^{m} d \theta_{h}^{n}=0$ and

$$
\begin{aligned}
& \left(\theta_{g}^{m}\right)_{(1)}\left(\theta_{h}^{n}\right)_{(1)} \otimes\left(\theta_{g}^{m}\right)_{(2)} d\left(\theta_{h}^{n}\right)_{(2)}=0, \\
& \left(\theta_{g}^{m}\right)_{(1)} d\left(\theta_{h}^{n}\right)_{(1)} \otimes\left(\theta_{g}^{m}\right)_{(2)}\left(\theta_{h}^{n}\right)_{(2)}=0 .
\end{aligned}
$$

Proof. Let $((g, m),(h, n)) \in B_{\Gamma}$. The statement that $\theta_{g}^{m} d \theta_{h}^{n}=0$ follows directly from the definition of $B_{\Gamma}$. We now prove (4.15). The proof of (4.16) is similar so we omit it.

Since $B_{\Gamma}$ is bicovariant, the element $((g, m),(h, n)) \in B_{\Gamma}$ falls into one of the following three cases:

case 1: $m \neq n$,

case 2: $m=n$ and $g^{-1} h \notin G_{m}$,

case 3: $m=n$ and $g^{-1} h \in G_{m}-\{e\}$.

For case 1, we have

$$
\left(\theta_{g}^{m}\right)_{(1)}\left(\theta_{h}^{n}\right)_{(1)} \otimes\left(\theta_{g}^{m}\right)_{(2)} d\left(\theta_{h}^{n}\right)_{(2)}=\sum_{x, y \in G} \theta_{g x^{-1}}^{x m} \theta_{h y^{-1}}^{y n} \otimes \theta_{x}^{m} d \theta_{y}^{n}=0 .
$$

Note that the last equality follows from the fact that $\theta_{x}^{m} d \theta_{y}^{n}=0$ for all $x, y \in G$ since $((x, m),(y, n)) \in B_{\Gamma}$ for $m \neq n$. For case 2 , we have

$$
\begin{aligned}
\left(\theta_{g}^{m}\right)_{(1)}\left(\theta_{h}^{m}\right)_{(1)} \otimes\left(\theta_{g}^{m}\right)_{(2)} d\left(\theta_{h}^{m}\right)_{(2)} & =\sum_{x, y \in G} \theta_{g x^{-1}}^{x m} \theta_{h y^{-1}}^{y m} \otimes \theta_{x}^{m} d \theta_{y}^{m} \\
& =\sum_{x, y \in G} \delta_{x m, y m} \delta_{g x^{-1}, h y^{-1}} \theta_{g x^{-1}}^{x m} \otimes \theta_{x}^{m} d \theta_{y}^{m} \\
& =\sum_{x^{-1} y \in G_{m}} \delta_{g^{-1} h, x^{-1} y} \theta_{g x^{-1}}^{x m} \otimes \theta_{x}^{m} d \theta_{y}^{m} \\
& =0
\end{aligned}
$$

since $g^{-1} h \notin G_{m}$ for case 2. Lastly, for case 3, we have $h=g k$ for some $k \in G_{m}-\{e\}$. Then

$$
\begin{aligned}
\left(\theta_{g}^{m}\right)_{(1)}\left(\theta_{g k}^{m}\right)_{(1)} \otimes\left(\theta_{g}^{m}\right)_{(2)} d\left(\theta_{g k}^{m}\right)_{(2)} & =\sum_{x, y \in G} \theta_{g x^{-1}}^{x m} \theta_{g k y^{-1}}^{y m} \otimes \theta_{x}^{m} d \theta_{y}^{m} \\
& =\sum_{x, y \in G} \delta_{x m, y m} \delta_{g x^{-1}, g k y^{-1}} \theta_{g x^{-1}}^{x m} \otimes \theta_{x}^{m} d \theta_{y}^{m} \\
& =\sum_{x \in G} \theta_{g x^{-1}}^{x m} \otimes \theta_{x}^{m} d \theta_{x k}^{m} \\
& =0
\end{aligned}
$$

where the last equality follows from condition (3) of Definition 4.1. Specifically, since $((g, m),(g k, m)) \in B_{\Gamma}$, we have $((x, m),(x k, m)) \in B_{\Gamma}$ for all 
$x \in G$, which in turn implies that $\theta_{x}^{m} d \theta_{x k}^{m}=0$ for all $x \in G$. This proves $(4.15)$.

Proposition 4.7. Let $(\Gamma, d)$ be a FODC over $\mathbb{k}(G \ltimes M)$ such that $B_{\Gamma}$ is bicovariant in the sense of Definition 4.1. Then $(\Gamma, d)$ is bicovariant.

Proof. Let $(\Gamma, d)$ be a FODC over $\mathbb{k}(G \ltimes M)$ such that $B_{\Gamma}$ is bicovariant. Let

$$
\widehat{E}_{u}:=\left\{\theta_{g}^{m} d_{u} \theta_{h}^{n} \mid((g, m),(h, n)) \in E_{u}\right\}
$$

and

$$
\widehat{B}_{\Gamma}:=\left\{\theta_{g}^{m} d_{u} \theta_{h}^{n} \mid((g, m),(h, n)) \in B_{\Gamma}\right\} \subset \widehat{E}_{u} .
$$

By Proposition 3.1, $\widehat{E}_{u}$ is a vector space basis of $\Omega_{u}^{1}$. Let

$$
\bar{\Delta}_{L}: \Omega_{u}^{1} \rightarrow \mathbb{k}(G \ltimes M) \otimes \Omega_{u}^{1}, \quad \bar{\Delta}_{R}: \Omega_{u}^{1} \rightarrow \Omega_{u}^{1} \otimes \mathbb{k}(G \ltimes M)
$$

be the linear maps whose values on the basis $\widehat{E}_{u}$ is as follows:

(a) for $\theta_{g}^{m} d_{u} \theta_{h}^{m} \in \widehat{E}_{u}-\widehat{B}_{\Gamma}$,

$$
\begin{aligned}
\bar{\Delta}_{L}\left(\theta_{g}^{m} d_{u} \theta_{h}^{m}\right) & =\left(\theta_{g}^{m}\right)_{(1)}\left(\theta_{h}^{m}\right)_{(1)} \otimes\left(\theta_{g}^{m}\right)_{(2)} d_{u}\left(\theta_{h}^{m}\right)_{(2)} \\
& =\sum_{x \in G} \theta_{g x^{-1}}^{x m} \otimes \theta_{x}^{m} d_{u} \theta_{x g^{-1} h}^{m}, \\
\bar{\Delta}_{R}\left(\theta_{g}^{m} d_{u} \theta_{h}^{m}\right) & =\left(\theta_{g}^{m}\right)_{(1)} d_{u}\left(\theta_{h}^{m}\right)_{(1)} \otimes\left(\theta_{g}^{m}\right)_{(2)}\left(\theta_{h}^{m}\right)_{(2)} \\
& =\sum_{x \in G} \theta_{g x^{-1}}^{x m} d_{u} \theta_{h x^{-1}}^{x m} \otimes \theta_{x}^{m}
\end{aligned}
$$

(b) $\left.\bar{\Delta}_{L}\right|_{\widehat{B}_{\Gamma}} \equiv 0$ and $\left.\bar{\Delta}_{R}\right|_{\widehat{B}_{\Gamma}} \equiv 0$.

Let $\psi_{\Gamma}:\left(\Omega_{u}^{1}, d_{u}\right) \rightarrow(\Gamma, d)$ be the natural morphism of FODCs given by Theorem 2.12. Since ker $\psi_{\Gamma}=\operatorname{span}_{\mathbb{k}} \widehat{B}_{\Gamma}$ and

$$
\operatorname{span}_{\mathbb{k}} \widehat{B}_{\Gamma} \subset \operatorname{ker} \bar{\Delta}_{L}, \quad \operatorname{span}_{\mathbb{k}} \widehat{B}_{\Gamma} \subset \operatorname{ker} \bar{\Delta}_{R},
$$

we see that $\bar{\Delta}_{L}$ and $\bar{\Delta}_{R}$ induce linear maps

$$
\widetilde{\Delta}_{L}: \Gamma \rightarrow \mathbb{k}(G \ltimes M) \otimes \Omega_{u}^{1}, \quad \widetilde{\Delta}_{R}: \Gamma \rightarrow \Omega_{u}^{1} \otimes \mathbb{k}(G \ltimes M)
$$

which are defined by the conditions

$$
\bar{\Delta}_{L}=\widetilde{\Delta}_{L} \circ \psi_{\Gamma}, \quad \bar{\Delta}_{R}=\widetilde{\Delta}_{R} \circ \psi_{\Gamma} .
$$

Now define

$$
\Delta_{L}: \Gamma \rightarrow \mathbb{k}(G \ltimes M) \otimes \Gamma, \quad \Delta_{R}: \Gamma \rightarrow \Gamma \otimes \mathbb{k}(G \ltimes M)
$$

by

$$
\Delta_{L}:=\left(i d \otimes \psi_{\Gamma}\right) \circ \widetilde{\Delta}_{L}, \quad \Delta_{R}:=\left(\psi_{\Gamma} \otimes i d\right) \circ \widetilde{\Delta}_{R}
$$

We now verify that $\Delta_{L}$ is a covariant left coaction. (The proof that $\Delta_{R}$ is a covariant right coaction is similar so we omit it.) To show that $\Delta_{L}$ satisfies the covariance condition (2.4), it suffices by Lemma 4.4 to show that $\Delta_{L}$ 
satisfies the covariance condition on all elements of the form $\theta_{g}^{m} d \theta_{h}^{n}$ where $\theta_{g}^{m} \neq \theta_{h}^{n}$. The set of all such elements is the image of $\widehat{E}_{u}$ under $\psi_{\Gamma}$. For $\theta_{g}^{m} d \theta_{h}^{m} \in \psi_{\Gamma}\left(\widehat{E}_{u}-\widehat{B}_{\Gamma}\right)$, we have

$$
\begin{aligned}
\Delta_{L}\left(\theta_{g}^{m} d \theta_{h}^{m}\right) & =\left(i d \otimes \psi_{\Gamma}\right) \circ \widetilde{\Delta}_{L}\left(\theta_{g}^{m} d \theta_{h}^{m}\right) \\
& =\left(i d \otimes \psi_{\Gamma}\right) \circ \bar{\Delta}_{L}\left(\theta_{g}^{m} d_{u} \theta_{h}^{m}\right) \\
& =\left(i d \otimes \psi_{\Gamma}\right)\left(\left(\theta_{g}^{m}\right)_{(1)}\left(\theta_{h}^{m}\right)_{(1)} \otimes\left(\theta_{g}^{m}\right)_{(2)} d_{u}\left(\theta_{h}^{m}\right)_{(2)}\right) \\
& =\left(\theta_{g}^{m}\right)_{(1)}\left(\theta_{h}^{m}\right)_{(1)} \otimes\left(\theta_{g}^{m}\right)_{(2)} d\left(\theta_{h}^{m}\right)_{(2)},
\end{aligned}
$$

where the third equality follows from the definition of $\bar{\Delta}_{L}$. Now let $\theta_{g}^{m} d_{u} \theta_{h}^{n} \in$ $\widehat{B}_{\Gamma}$. Then $\theta_{g}^{m} d \theta_{h}^{n}=\psi_{\Gamma}\left(\theta_{g}^{m} d_{u} \theta_{h}^{n}\right)=0$ and

$$
\Delta_{L}\left(\theta_{g}^{m} d \theta_{h}^{n}\right)=0=\left(\theta_{g}^{m}\right)_{(1)}\left(\theta_{h}^{n}\right)_{(1)} \otimes\left(\theta_{g}^{m}\right)_{(2)} d\left(\theta_{h}^{n}\right)_{(2)}
$$

by Lemma 4.6. With the completion of this step, we have shown that for all $\theta_{g}^{m} \neq \theta_{h}^{n}$, we have

$$
\Delta_{L}\left(\theta_{g}^{m} d \theta_{h}^{n}\right)=\left(\theta_{g}^{m}\right)_{(1)}\left(\theta_{h}^{n}\right)_{(1)} \otimes\left(\theta_{g}^{m}\right)_{(2)} d\left(\theta_{h}^{n}\right)_{(2)} .
$$

Lemma 4.4 now implies that $\Delta_{L}$ satisfies the covariance condition (2.4).

We now quickly verify that $\Delta_{L}$ is indeed a left coaction of $\mathbb{k}(G \ltimes M)$. Specifically, we must verify that

$$
(\Delta \otimes i d) \circ \Delta_{L}=\left(i d \otimes \Delta_{L}\right) \circ \Delta_{L}, \quad(\varepsilon \otimes i d) \circ \Delta_{L}=i d .
$$

The first left coaction axiom follows immediately from the multiplicativity and coassociativity of $\Delta$ and the fact that $\Delta_{L}$ satisfies the covariance condition (2.4). For the second coaction axiom, it suffices to verify it on the elements of $\psi_{\Gamma}\left(\widehat{E}_{u}-\widehat{B}_{\Gamma}\right)$ which is a vector space basis for $\Gamma$. The elements of $\psi_{\Gamma}\left(\widehat{E}_{u}-\widehat{B}_{\Gamma}\right)$ are of the form $\theta_{g}^{m} d \theta_{g k}^{m}$ where $m \in M, g \in G$, and $k \in G_{m}-\{e\}$. Using the covariance of $\Delta_{L}$, we have

$$
\begin{aligned}
(\varepsilon \otimes i d) \circ \Delta_{L}\left(\theta_{g}^{m} d \theta_{g k}^{m}\right) & =\varepsilon\left(\left(\theta_{g}^{m}\right)_{(1)}\left(\theta_{g k}^{m}\right)_{(1)}\right)\left(\theta_{g}^{m}\right)_{(2)} d\left(\theta_{g k}^{m}\right)_{(2)} \\
& =\sum_{x \in G} \varepsilon\left(\theta_{g x^{-1}}^{x m}\right) \theta_{x}^{m} d \theta_{x k}^{m}=\sum_{x \in G} \delta_{g x^{-1}, e} \theta_{x}^{m} d \theta_{x k}^{m} \\
& =\theta_{g}^{m} d \theta_{g k}^{m} .
\end{aligned}
$$

Lastly, since $\Delta_{L}$ and $\Delta_{R}$ satisfy the covariance conditions (2.4) and (2.5) respectively, it follows that $\Delta_{L}$ and $\Delta_{R}$ are compatible, that is, they satisfy

$$
\left(i d \otimes \Delta_{R}\right) \circ \Delta_{L}=\left(\Delta_{L} \otimes i d\right) \circ \Delta_{R} .
$$

The proof of Theorem 4.2 now follows from Propositions 4.5 and 4.7. We conclude this paper with the following classification result. 
Theorem 4.8. For $m \in M$, let $\bar{G}_{m}:=G_{m}-\{e\}$. Define a left $G$ action on $\mathcal{J}(M, G):=\bigcup_{m \in M} \bar{G}_{m} \times\{m\}$ by $x \cdot(k, m):=\left(x k x^{-1}, x m\right)$ for all $x \in G, m \in M$, and $k \in \bar{G}_{m}$. Then there is a one-to-one correspondence between isomorphism classes of bicovariant FODC over $\mathbb{k}(G \ltimes M)$ and subsets of $\mathcal{J}(M, G) / G$.

Proof. Theorem 4.2 and the proof of Theorem 3.4 imply that there is a one-to-one correspondence between isomorphism classes of bicovariant FODC over $\mathbb{k}(G \ltimes M)$ and subsets $E$ of $E_{u}$ for which $E_{u}-E$ is bicovariant in the sense of Definition 4.1. Worded differently, we have a one-to-one correspondence between isomorphism classes of bicovariant FODC over $\mathbb{k}(G \ltimes M)$ and subsets $B$ of $E_{u}$ which are bicovariant.

For any subset $B$ of $E_{u}$, define

$$
B^{\star}:=B \cap\left\{((g, m),(g k, m)) \mid g \in G, m \in M, k \in \bar{G}_{m}\right\} .
$$

It follows immediately from Definition 4.1 that two bicovariant subsets $B_{1}$ and $B_{2}$ are equal if and only if $B_{1}^{\star}$ and $B_{2}^{\star}$ are equal.

Let $\pi: \mathcal{J}(M, G) \rightarrow \mathcal{J}(M, G) / G$ denote the quotient map and define $\varphi: E_{u}^{\star} \rightarrow \mathcal{J}(M, G)$ by

$$
\varphi(((g, m),(g k, m))):=\left(g^{-1}(g k), m\right)=(k, m)
$$

and let $\bar{\varphi}=\pi \circ \varphi$. Now suppose that $B_{1}$ and $B_{2}$ are bicovariant subsets such that

$$
\bar{\varphi}\left(B_{1}^{\star}\right)=\bar{\varphi}\left(B_{2}^{\star}\right) .
$$

Let $((g, m),(g k, m)) \in B_{1}^{\star}$. Since

$$
\bar{\varphi}((g, m),(g k, m))=\pi(k, m) \in \bar{\varphi}\left(B_{2}^{\star}\right),
$$

it follows that $\left(x k x^{-1}, x m\right) \in \varphi\left(B_{2}^{\star}\right)$ for some $x \in G$. Hence,

$$
\left((h, x m),\left(h\left(x k x^{-1}\right), x m\right)\right) \in B_{2}^{\star} \subset B_{2}
$$

for some $h \in G$. Condition (3) of Definition 4.1 implies that

$$
\left((a, y x m),\left(a\left(y x k x^{-1} y^{-1}\right), y x m\right)\right) \in B_{2}^{\star}
$$

for all $a, y \in G$, where we note that $y x k x^{-1} y^{-1} \in \bar{G}_{y x m}$. Setting $y=x^{-1}$ and $a=g$, we have $((g, m),(g k, m)) \in B_{2}^{\star}$. Hence, $B_{1}^{\star} \subset B_{2}^{\star}$. The same argument also gives $B_{1}^{\star} \supset B_{2}^{\star}$. This shows that

$$
B_{1}^{\star}=B_{2}^{\star} \Leftrightarrow \bar{\varphi}\left(B_{1}^{\star}\right)=\bar{\varphi}\left(B_{2}^{\star}\right) .
$$

Let $\mathrm{BiCov}$ denote the set of bicovariant subsets of $E_{u}$ and let $\operatorname{Sub}(\mathcal{J}(M, G) / G)$ denote the set of all subsets of $\mathcal{J}(M, G) / G$. Define

$$
\Phi: \operatorname{BiCov} \rightarrow \operatorname{Sub}(\mathcal{J}(M, G) / G)
$$

by $\Phi(B):=\bar{\varphi}\left(B^{\star}\right)$. The above argument shows that $\Phi$ is injective. To see that $\Phi$ is also surjective, let $X$ be a subset of $\mathcal{J}(M, G) / G$. Now let $Q \subset E_{u}$ 
be the subset consisting of all elements of $E_{u}$ which satisfy conditions (1) and (2) of Definition 4.1. Now let

$$
B=Q \cup(\bar{\varphi})^{-1}(X) .
$$

Observe that if $((g, m),(g k, m)) \in B$ for some $g \in G, m \in M$, and $k \in \bar{G}_{m}$, then $((g, m),(g k, m))$ must lie in $(\bar{\varphi})^{-1}(X)$. This implies that $\pi(k, m) \in X$. Since $\pi(k, m)=\pi\left(y k y^{-1}, y m\right)$ for all $y \in G$, it follows that

$$
\left((h, y m),\left(h y k y^{-1}, y m\right)\right) \in(\bar{\varphi})^{-1}(X) \subset B \quad \forall h, y \in G .
$$

Hence, $B$ satisfies condition (3) of Definition 4.1. In particular, $B$ is a bicovariant subset. Since $\varphi: E_{u}^{\star} \rightarrow \mathcal{J}(M, G)$ is surjective, we also have

$$
\Phi(B):=\bar{\varphi}\left(B^{\star}\right)=\bar{\varphi}\left((\bar{\varphi})^{-1}(X)\right)=X .
$$

REMARK 4.9. In the special case when $M=\{m\}$ is a point, we have $\mathbb{k}(G \ltimes M)=\mathbb{k}(G), \bar{G}_{m}=\bar{G}:=G-\{e\}, \mathcal{J}(M, G)=\bar{G}$, and $\mathcal{J}(M, G) / G$ is equal to the set of non-trivial conjugacy classes of $G$. Consequently, when $M$ is a point, Theorem 4.8 reduces to the well known fact that the isomorphism classes of bicovariant FODC over $\mathbb{k}(G)$ are in one to one correspondence with subsets of the set of non-identity conjugacy classes of $G$.

Example 4.10. Consider the set $M=\{1,2,3\}$ and let the symmetric group

$$
S_{3}=\{e,(1,2),(1,3),(2,3),(1,2,3),(1,3,2)\}
$$

act naturally on $M$. Then

$$
\begin{aligned}
\mathcal{J}\left(M, S_{3}\right) & =\bigcup_{i=1}^{3}{\overline{\left(S_{3}\right)_{i}}} \times\{i\} \\
& =\{(2,3)\} \times\{1\} \cup\{(1,3)\} \times\{2\} \cup\{(1,2)\} \times\{3\} .
\end{aligned}
$$

It is easy to see that $\left|\mathcal{J}\left(M, S_{3}\right) / S_{3}\right|=1$. Theorem 4.8 implies that (up to isomorphism) there are exactly two bicovariant FODCs over $\mathbb{k}\left(S_{3} \ltimes M\right)$. One, of course, is the trivial zero-dimensional FODC over $\mathbb{k}\left(S_{3} \ltimes M\right)$. Let us denote the non-trivial bicovariant FODC over $\mathbb{k}\left(S_{3} \ltimes M\right)$ by $(\Gamma, d)$. Also, let

$$
E_{u}:=\left\{((\alpha, i),(\beta, j)) \mid(\alpha, i) \neq(\beta, j), \alpha, \beta \in S_{3}, i, j \in M\right\}
$$

and let $\alpha_{1}:=(2,3), \alpha_{2}:=(1,3)$, and $\alpha_{3}:=(1,2)$. The FODC $(\Gamma, d)$ corresponds to the directed graph whose vertices are the elements of the set $S_{3} \times M$ and whose directed edges are given by the set

$$
E_{\Gamma}=\bigcup_{i=1}^{3}\left\{\left((\sigma, i),\left(\sigma \alpha_{i}, i\right)\right) \mid \sigma \in S_{3}\right\} .
$$


From this, we have $\operatorname{dim} \Gamma=\left|E_{\Gamma}\right|=18$. One easily sees that $E_{\Gamma}$ is the only non-empty subset of $E_{u}$ such that $E_{u}-E_{\Gamma}$ is bicovariant in the sense of Definition 4.1.

ACKNowledgements.

The author would like to thank the referees for their careful reading of this paper and for their valuable and constructive comments.

\section{REFERENCES}

[1] L. Abrams, Two dimensional topological quantum field theories and Frobenius algebras, J. Knot Theory Ramifications 5 (1996), 569-587.

[2] E. Batista, Noncommutative geometry: a quantum group approach, Mat. Contemp. 28 (2005), 63-90.

[3] G. Böhm, F. Nill and K. Szlachányi, Weak Hopf algebras. I. Integral theory and $C^{*}$ structure, J. Algebra 221 (1999), 385-438.

[4] T. Brzeziński and S. Majid Quantum group gauge theory on quantum spaces, Comm. Math. Phys. 157 (1993), 591-638.

[5] K. Bresser, F. Müller-Hoissen, A. Dimakis and A. Sitarz, Non-commutative geometry of finite groups, J. Physics A 29 (1996), 2705-2735.

[6] J. Chen and S. Wang, Differential calculi on quantum groupoids, Comm. Algebra 36 (2008), 3792-3819.

[7] A. Connes, Noncommutative differential geometry, Inst. Hautes Études Sci. Publ. Math. 62 (1985), 275-360.

[8] A. Connes, Noncommutative geometry, Academic Press, Inc., San Diego, 1994.

[9] A. Dimakis and F. Müller-Hoissen, Discrete differential calculus, graphs, topologies and gauge theory, J. Math. Physics 35 (1994), 6703-6735.

[10] B. Fantechi and L. Goettsche, Orbifold cohomology for global quotients, Duke Math. J. 117 (2003), 197-227.

[11] X. Gomez and S. Majid, Noncommutative cohomology and electromagnetism on $\mathbb{C}_{q}\left[S L_{2}\right]$ at roots of unity, Lett. Math. Phys. 60 (2002), 221-237.

[12] T. Jarvis, R. Kaufmann and T. Kimura, Stringy K-theory and the Chern character, Invent. Math. 168 (2007), 23-81.

[13] R. Kaufmann, Orbifolding Frobenius algebras, Internat. J. Math. 14 (2003), 573-619.

[14] R. Kaufmann, Global stringy orbifold cohomology, K-theory and de Rham theory, Lett. Math. Phys. 94 (2010), 165-195.

[15] G. Landi, An introduction to noncommutative spaces and their geometries, Springer, 1997.

[16] J. Madore, An introduction to noncommutative differential geometry and its physical applications, 2nd. Ed. Cambridge University Press, Cambridge, 1999.

[17] S. Majid Non-commutative differential geometry, in: Analysis and mathematical physics, World Sci. Publ., Hackensack, 2017, 139-176.

[18] S. Majid, Riemannian geometry of quantum groups and finite groups with nonuniversal differentials, Comm. Math. Phys. 225 (2002), 131-170.

[19] J. Nestruev, Smooth manifolds and observables, Springer-Verlag, New York, 2003.

[20] S. B. Sontz, Principal bundles. The quantum case, Springer, 2015.

[21] M. Sweedler, Hopf algebras, W. A. Benjamin, Inc., New York, 1969.

[22] V. Turaev, Homotopy field theory in dimension 2 and group-algebras, arXiv.org:math/9910010, (1999).

[23] S. L. Woronowicz, Differential calculus on compact matrix pseudogroups (quantum groups), Comm. Math. Phys. 122 (1989), 125-170. 
BICOVARIANT DIFFERENTIAL CALCULI FOR FINITE GLOBAL QUOTIENTS 499

[24] H. Zhu, S. Wang and J. Chen Bicovariant differential calculi on a weak Hopf algebra, Taiwanese J. Math. 18 (2014), 1679-1712.

D. Pham

Department of Mathematics \& Computer Science

Queensborough C. College, City University of New York

Bayside, NY 11364

USA

E-mail: dpham90@gmail.com

Received: 28.10.2018.

Revised: 10.6.2019. 